\title{
Relating Diffuse Interstellar Band Strengths to Line of Sight Properties
}

\author{
H. Rashedi ${ }^{1}$, J. Cami ${ }^{1,2}$, N. L. J. Cox ${ }^{3}$ and H. van Winckel ${ }^{3}$ \\ ${ }^{1}$ Department of Physics \& Astronomy, University of Western Ontario, \\ London, ON N6A 3K7, Canada \\ ${ }^{2}$ SETI Institute, 189 Bernardo Ave, Suite 100, Mountain View, CA 94043, USA \\ ${ }^{3}$ Institute for Astronomy, KU Leuven, Celestijnenlaan 200D, B-3001, Leuven, Belgium
}

\begin{abstract}
We present a preliminary analysis of a set of optical (3800-8800 $\AA$ ) high resolution $(R=80,000)$ spectra for 69 diffuse interstellar band targets. We carried out a sensitive search for interstellar features in the wavelength range $8470-8740 \AA$ that will be covered by the upcoming GAIA mission. We also investigate how the $\lambda 8620 \AA$ DIB strength varies as a function of other interstellar parameters (other DIBs, $E(B-V)$ and atomic and molecular column densities).
\end{abstract}

Keywords. ISM:molecules; ISM:lines and bands

\section{Introduction \& Motivation}

As part of the main science objectives for the upcoming GAIA mission (Perryman et al. 2001), its Radial Velocity Spectrometer (RVS, Katz et al. 2004) will obtain spectra in the wavelength range 848-874 $\mathrm{nm}$ at a resolving power of $R \sim 11,500$ for 100-150 million stars in the Milky Way galaxy. This unprecedented data set offers a great serendipitous opportunity to study the physical and chemical conditions of the intervening interstellar medium (ISM). While several authors have discussed the presence of a few diffuse interstellar bands (DIBs) in this wavelength range (see e.g. Munari \& Castelli 2000; Wallerstein et al. 2007; Munari et al. 2008, and references therein), it is not clear how much information about the properties of the ISM can be extracted from the limited wavelength range covered by the GAIA/RVS.

Here, we present preliminary results of an observational study whose main goal is to investigate what ISM conditions can be reliably probed by the GAIA/RVS observations. The key objectives of this program are to $i$ ) make a detailed inventory of interstellar features in GAIA range by carrying out a sensitive survey; ii) compare the properties of these features to other line of sight parameters (e.g. reddening, strength of DIBs in other ranges,... ); and $i i i)$ investigate whether we can establish quantitative relations between DIBs in the GAIA range and these other line of sight parameters. If possible, this would allow to use GAIA/RVS observations to determine properties of the ISM that are not directly accessible from the GAIA/RVS wavelength range.

\section{Observations and data processing}

The sample of targets we discuss here consists of 69 typical DIB targets: O, B and early A spectral type stars with $E(B-V)=0.1$ to $E(B-V)=2.1$. We have also included two unreddened telluric and spectroscopic standards. All of our targets were observed with the High Efficiency and Resolution Mercator Echelle Spectrograph (HERMES, Raskin et al. 2011) mounted on the $1.2 \mathrm{~m}$ Mercator telescope. These observations cover the range $3800-8800 \AA$, at a spectral resolving power of $\mathrm{R} \sim 80,000$. The data were reduced using the 


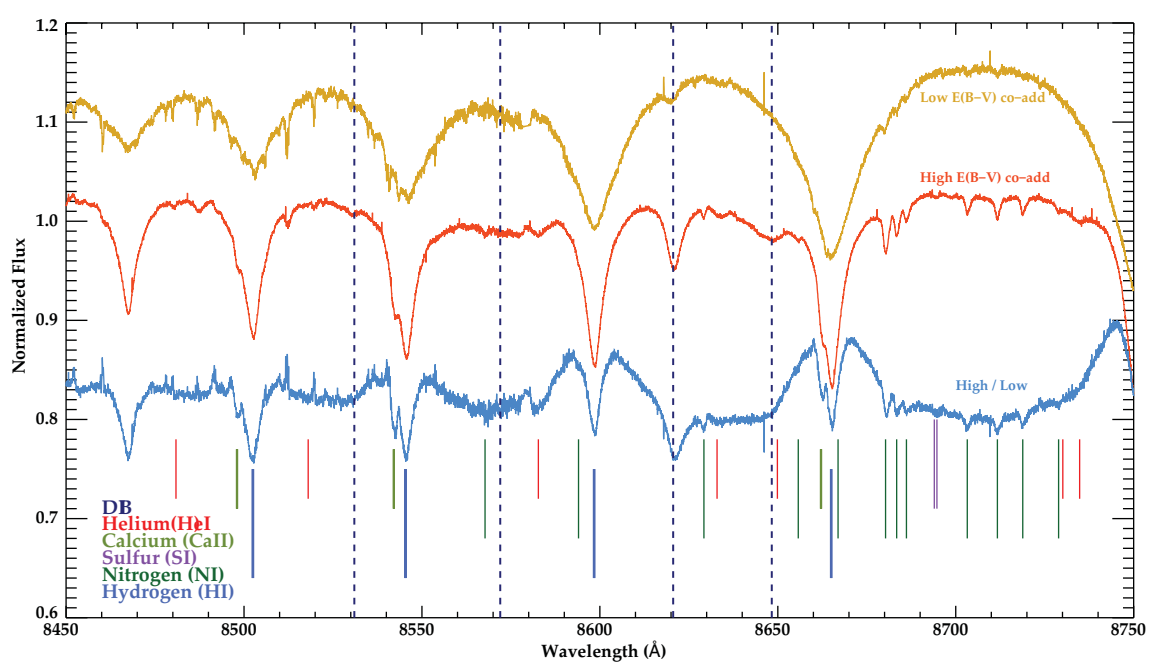

Figure 1. Two superspectra in the GAIA/RVS range: one comprised of 38 highly reddened targets with $E(B-V) \geqslant 0.5$ (middle; S/N 730) and a second one corresponding to 14 targets with $E(B-V)<0.25$ (top; $\mathrm{S} / \mathrm{N} \sim 320$ ). The ratio of these two spectra is shown at the bottom. Tick marks indicate the wavelengths of various stellar lines; dashed lines indicate known or suggested DIBs. Shortward of $8600 \AA$, many telluric features are present.

dedicated automated data reduction pipeline and radial velocity toolkit (HermesDRS) following a standard recipe that performs all required reduction steps and results in a fully resampled, single spectrum per observation.

\section{Interstellar Features in the GAIA range}

We cross-correlated our spectra with a model containing unresolved and unsaturated NaI D lines to determine interstellar cloud radial velocities. Stellar radial velocities were obtained from the SIMBAD database. We then restricted our spectra to the wavelength range $8470-8740 \AA$, and created GAIA "superspectra": spectra obtained by shifting the indvidiual observations to a specific rest frame and then co-adding a large number of spectra from different targets to increase the signal-to-noise $(\mathrm{S} / \mathrm{N})$ ratio (see also Bhatt et al., this volume). Here, we present two interstellar superspectra: one obtained from 38 targets with $E(B-V) \geqslant 0.50$, and another from 14 targets with $E(B-V)<0.25$. A comparison between these two superspectra better reveals broad DIBs (see Fig. 1).

Four DIBs have been previously suggested in this wavelength range. The well-known $8620 \AA$ DIB is clearly present in our reddened superspectrum, and is also quite strong in individual targets. Also the DIB at $8648.3 \AA$ shows up quite clearly in our spectra. This DIB was first suggested by Sanner et al. (1978) and later confirmed by other authors. A sharp DIB was suggested at $8530.7 \AA$ by Jenniskens \& Desert (1994), but this DIB was not confirmed by Galazutdinov et al. (2000), and also Munari et al. (2008) cast doubts on the presence of this DIB, pointing out likely contamination from an underlying HeI line. A weak feature does indeed show up in our reddened superspectrum, but it is hard to establish its nature. Finally, a feature at $8572 \AA$ was first tentatively proposed as a DIB, but later it was shown to be photospheric in nature. We do not see any trace of such a feature in our superspectra. No other interstellar absorption features are obvious from our comparison. However, the presence of many stellar and telluric lines makes it very difficult to conclusively decide on the presence of interstellar features. Future work 

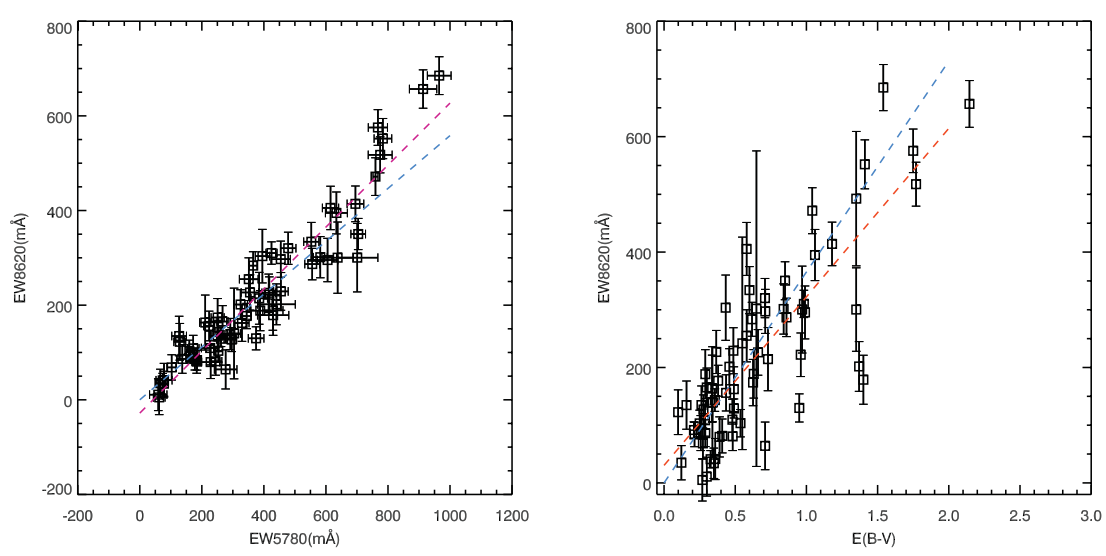

Figure 2. The very strong correlation between the $\lambda 8620$ and $\lambda 5780$ DIBs in our sample (left) and the correlation with $E(B-V)$ (right). For comparison, a least absolute deviation fit is shown in red, and a linear fit forced through the origin in blue.

will include fitting the stellar lines and removing the telluric features to obtain a "pure" interstellar spectrum.

\section{Relating the $\lambda 8620$ DIB strength to other parameters}

To relate the strength of the $\lambda 8620$ DIB to other DIBs and reddening, we measured the equivalent widths of 7 more strong and well-known DIBs $(\lambda \lambda 5780,5797,5850,6196$, $6270,6379$ and $6613 \AA)$. We confirm that the $\lambda 8620 \AA$ DIB correlates well with $E(B-V)$ (Pearson correlation coefficient 0.82 ), but shows an especially tight correlation with the strength of the $\lambda 5780 \AA$ DIB (correlation coefficient of 0.95 ; see Fig. 2). Correlations with other DIBs are not nearly as tight (correlation coefficients ranging from 0.59 to 0.86 ). Since the $\lambda 5780$ DIB in turn correlates very well with the column density of neutral $\mathrm{H}$ (Herbig 1993; Friedman et al. 2011), this could imply that the GAIA/RVS observations could be used to indirectly determine the neutral $\mathrm{H}$ content in millions of lines of sight.

\section{Acknowledgements}

HR and JC acknowledge support from an NSERC Discovery Grant. HR thanks the IAU for providing travel support to attend this symposium.

\section{References}

Friedman, S. D., York, D. G., McCall, B. J., et al. 2011, ApJ 727, 33

Galazutdinov, G. A., Musaev, F. A., Krełowski, J., \& Walker, G. A. H. 2000, PASP 112, 648

Herbig, G. H. 1993, ApJ 407, 142

Jenniskens, P. \& Desert, F. X. 1994, A\&AS 106, 39

Katz, D., Munari, U., Cropper, M., et al. 2004, MNRAS 354, 1223

Munari, U. \& Castelli, F. 2000, A\&AS 141, 141

Munari, U., Tomasella, L., Fiorucci, M., et al. 2008, A\&\&A 488, 969

Perryman, M. A. C., de Boer, K. S., Gilmore, G., et al. 2001, A\&A 369, 339

Raskin, G., van Winckel, H., Hensberge, H., et al. 2011, A $8 A$ 526, A69

Sanner, F., Snell, R., \& vanden Bout, P. 1978, ApJ 226, 460

Wallerstein, G., Sandstrom, K., \& Gredel, R. 2007, PASP 119, 1268 